\title{
Situational Awareness and Systems for Driver-Assistance
}

\author{
Hasan J. Alyamani \\ Department of Computing \\ VISOR Research Group \\ Macquarie University, Australia \\ hasan.alyamani@students.mq.edu.au
}

\author{
Manolya Kavakli \\ Department of Computing \\ VISOR Research Group \\ Macquarie University, Australia \\ manolya.kavakli@mq.edu.au
}

\begin{abstract}
Low level of driver's situation awareness (SA) and high level of cognitive load are considered as reasons of vehicle accidents. Cognitive load is higher when driving abroad because of unfamiliarity with differences in international traffic rules or vehicle configurations. This paper aims to objectively assess the driver's $S A$ when performing lane changing tasks under unfamiliar driving conditions. We conducted an experiment using a right-hand driving simulator and a left-hand simulated traffic scenario to collect the temporal information about SA such as time, location, and speed as well as lane changing errors. Overall, the participants show low SA in curved roads and road networks, but high SA in straight roads. The results state that speed does not affect the lane changing performance on straight roads and road networks but significantly affects the lane changing performance on curved roads. These findings can be used to design a SA system for driver-assistance in unfamiliar driving conditions considering drivers' cognitive load.
\end{abstract}

\section{Introduction}

Cognitive load refers to the total amount of mental effort in working memory. "Cognitive load theory has been designed to provide guidelines intended to assist in the presentation of information in a manner that encourages learner activities that optimize intellectual performance" [1]. Cognitive load increases, while drivers perform a secondary task such as talking or texting on the phone or while they drive in unfamiliar conditions. Traffic rules and vehicle configuration differ from one country to another. Therefore, international drivers might face difficulties, while driving under an Unfamiliar Driving Condition (UFDC). In this research, an UFDC refers to unfamiliar traffic rules (i.e. driving on the left-hand side of the road) for drivers from right-handed traffic regulations, and driving an unfamiliar vehicle configuration (i.e. a right-hand drive vehicle) for drivers who are familiar with driving a left-hand drive vehicle. This might contribute to an increase in the vehicle accidents and fatalities. For instance, international visitors who come from countries with a right-handed traffic system are more likely to contribute to the number of vehicle accidents in Australia [2] and New Zealand [3], since these countries follow the left-handed traffic system. Those international drivers face difficulties especially in keeping the vehicle to the left (in the left-handed traffic system) and cause head-on crashes [2,3]. Even worse, Australia might face this issue more in future as the number of international visitors from countries with opposite driving conditions, such as the USA, Canada and Middle East increase [4].

Driver-assistance systems aim to reduce the number of fatalities and the severity of traffic accidents. These systems support driver by either warning them or automating the control of the task the driver is going to perform [5]. To gain a more reliable system, researchers study the drivers' behavior of a certain driving scenario or conditions to understand how the drivers interact and then design the system accordingly. This research aims to explore the international drivers' behaviour when they perform lane change tasks under UFDC by answering the following questions:

RQ1: When does the driver make errors in lane changing tasks under an UFDC?

RQ2: Where does the driver make errors in lane changing tasks under an UFDC?

RQ3: Why does the driver make errors in lane changing tasks under an UFDC?

Answering these questions will help us in further studies to design a driver-assistance system for international drivers to safely drive in UFDC.

In this project, our goal is to address when, where, and why the driver's situation awareness (SA) is low in an UFDC and thus the feedback regarding important information should be presented to the driver in a system for driver-as sis tance. 


\section{Situation Awareness}

Situational Awareness (SA) is recognised as an important factor in the performance of individuals. SA involves both temporal and spatial components. Endsley [6] defined SA as "the perception of the elements in the environment within a volume of time and space, the comprehension of their meaning and the projection of their status in the near future". This concept is divided into three levels: (level 1) perception, (level 2) comprehension and (level 3) projection, as seen in Figure1. Individuals become more aware of their situation when they manage to apply SA levels. Also from the definition of SA, we can extract two important temporal components of SA, time and space [7]. Time refers to available time until some event happens or some action must be taken, whereas space refers to how far away the object is. In highly dynamic environments, such as driving, a third temporal component of SA is included, which is the dynamic aspect of real-world situation (e.g. speed). This component helps the drivers for example to keep themselves updated with the situation and thus allows them to do projection of future situation. For example, [8] states that the speed is negatively correlated with cognitive load. However, is this true for all aspects of roads, such as straight, curved, intersections and roundabouts? Overall, SA is influenced by the goals, and other aspects of individual's cognition, including long-term memory, information processing mechanisms and automaticity [9] (see Figure 1).

SA can be measured by several methods, objective, subjective, and performance measurements. Objective measurements are considered as direct assessments of SA. They collect the participant's perceptual situation of a certain event and compare it with what is actually going on in order to assess the accuracy of the participant's SA at a certain point of time. Situation Awareness Global Assessment Technique (SAGAT) [6], and WOMBAT Situational Awareness and Stress Tolerance Test mostly (WOMBAT) [10] are examples of objective SA measurements.

Subjective measurements are another example of direct assessments of SA. They assess the individual's SA using an anchor scale (e.g. Participant Situation Awareness Questionnaire (PSAQ) [10] and the Situation Awareness Rating Technique (SART) [12]).

Performance measurements are considered as indirect SA assessment. This kind of measurements are based on the assumption of better performance means better SA. Thus, they infer individual's SA from the task performance outcomes (e.g. the accuracy of the response or the number of errors committed when performing a task).
Objective measurements feature the ability of objectively collecting the related data without disrupting task performance. In driving, which is already associated with high cognitive load, objective measurements might be the most useful and safe SA as sessment technique, as they neither disturb the driver nor increase the driver's cognitive load.

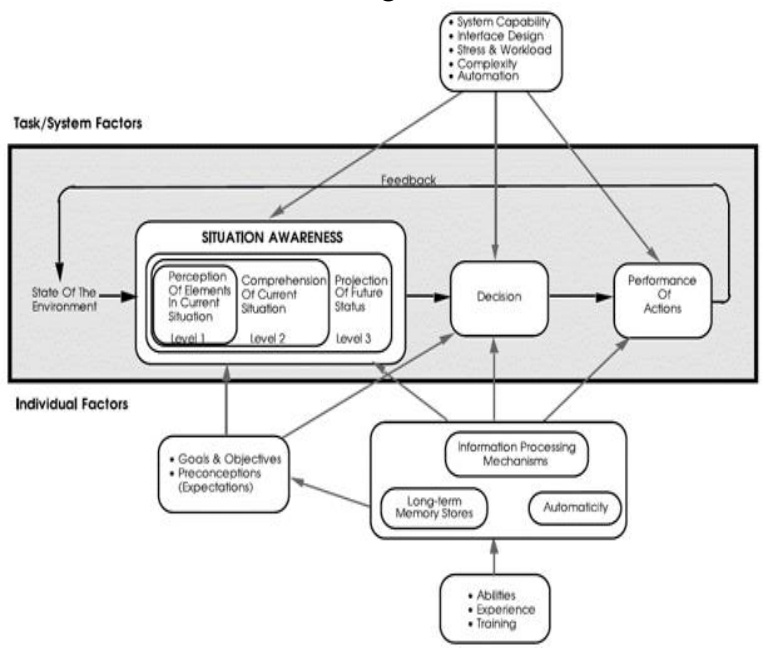

Figure 1. Model of SA in a dynamic decision making environment [25].

Driving is an example of a complex task which requires a set of skills, including perceptual, cognitive and motor skills [13]. Perceptual skills require levels 1 , 2 and 3 of SA. Cognitive processes send the output of SA to motor processes as proper commands to perform a set of physical actions. However, high cognitive load hinders adequate driving skills. Low level of SA in driving tasks as well as high cognitive load may contribute to the number of car accidents [14, 15]. Drivers then might ignore or pay too little attention to important and useful information. This may decrease the level of driver's SA. Cognitive processes might be delayed due to high cognitive load. Thus, the physical reaction is affected as a result of it.

The ability to scan and capture only the most important information is crucial to reduce cognitive load. It is important to measure the drivers' SA, when designing a SA system. However, to the best of our knowledge, there are no studies so far focusing on components of SA (time, location and speed) when designing systems for driver-assistance, particularly in high cognitive load conditions, such as unfamiliar driving conditions (UFDC).

Driving simulators are used in many studies including studies that aim to explore driving behavior whilst performing complex driving tasks which are usually as sociated with high cognitive load. Generally, driving simulators test the driving skills and specifically motor skills in a safe driving environment. 
In driving simulators, it is possible to mount additional hardware or technology (e.g. an eye tracker $[16,17]$ and electroencephalogram [18]) to test perceptual and cognitive skills in driving. Driving simulators also provide facilities to collect a large amount of data related to driving tasks. The collected data may help develop or explore new tools to support driving tasks (e.g. a personal investigation device [19] and electronic stability control (ESC) [20]). Driving simulators also offer a safer [21] and more flexible driver training environment [22]. They provide training under irregular weather conditions that the driver does not regularly experience while driving. In addition, driving simulators are used to investigate and analyse the driver's behaviour [23, 24]. However, there are not many studies that focus on SA in driving simulators to enhance the skills in lane changing tasks, particularly in high cognitive load conditions (i.e. an UFDC).

\section{Situation Awareness in Driving Domain}

In general, driving tasks, such as lane-keeping, avoiding hazards, entering or exiting from roundabouts, and parking mainly require a set of complex skills which involve, perceptual, cognitive and motor processes [13]. Perceptual processes include capturing surrounding information using audio and visual channels, comprehending and projecting it for near future. Cognitive processes, such as reasoning and decision making collect the captured information and then process it in order to send the appropriate motor commands to motor processes. Motor skills, such as steering, speeding and braking, receive and perform these commands. Perceptual processes in driving tasks involve the three levels of $\mathrm{SA}$, perception, comprehension and projection.

In the driving domain, $\mathrm{SA}$ is defined as "understanding the relationship between the driver's goal, the vehicle states, the road environment and infrastructure, and the behaviour of other road users at any moment in time" [26]. SA refers to recognizing the drivers' awareness of 'what is going on' [27]. However, high cognitive load influences driving skills and the level of SA. Decreasing SA and increasing the driver's cognitive load concurrently may cause road accidents $[14,15]$. A desired action of safely driving a vehicle might be miss-executed due to high cognitive load. The driver in this case is less likely to concentrate on the road straight ahead and more likely to ignore the peripheral vision [19] and thus is not able to perceive hazards and/or changes in the traffic situation [24]. Increasing cognitive load on cognitive processes results in delaying and interrupting processing of captured information and therefore issuing the appropriate reaction in a longer time [28]. Some studies also found that cognitive load affects motor skills, such as steering control [29], acceleration and deceleration [30]. While some systems aim to improve the driver SA, they may ignore the driver's cognitive load. These should be taken into account when designing a system for driver-as sistance.

\section{Driving under Unfamiliar Driving Conditions}

Traffic system mainly consists of three interactive factors: road users (e.g. drivers, passengers, pedestrians), the road environment (e.g. traffic rules, road aspects, weather) and vehicles on road, which might differ by their configurations (e.g. left-/righthanded vehicles), size and type [31]. In some circumstances, inappropriate interaction among these factors will result in traffic incidents.

A driver is an instance of 'road users' who plays a critical role in the traffic system that is the subject of the driving task. Drivers become unfamiliar with the traffic system when they interact with other unfamiliar traffic system components, namely environment and vehicle. In this research, the term unfamiliar driving condition (UFDC) refers to an unfamiliar driving environment (i.e. left-handed traffic rules) when using an unfamiliar vehicle (i.e. a right-handed vehicle). Some studies focus on the driver behavior and performance in order to explore and understand the psychological mechanisms of human behavior in various driving conditions. Driving behavior refers to the way of the driver interacts with the other traffic components [32], while the driving performance refers to the assessed accuracy or observed driving errors associated with the performance of a certain driving task.

Saito et al. [23] use a driving simulator to draw comparisons between the lane-keeping task of drivers in familiar and unfamiliar vehicle configurations. In particular, they study drivers coming from a right-hand drive vehicle background (i.e. Japan) who drive in both a right-hand and a left-hand drive vehicle and drive under familiar driving traffic rules (i.e. left-hand traffic). The result of Saito et al. [23] study shows that the ratio of lane departure with an unfamiliar left-hand drive vehicle configuration is higher than when driving a familiar right-hand drive vehicle configuration. More interestingly, the researchers of this study found that drivers with more experience in driving a right-hand drive vehicle might be highly influenced by driving an unfamiliar left-hand driving vehicle configuration. Thus, driving experience using a familiar vehicle 
configuration does not necessarily result in a safer drive in an unfamiliar vehicle configuration.

Therefore, it is important to improve a driver's SA to perform lane changing when driving under an UFDC using a driving simulator even if the driver is experienced. Measuring the driver SA helps us to know exactly when and where the drivers needs a driverassistance system without increasing the driver cognitive load.

\section{Research Model}

Generally, driving tasks require combining realtime information with prior knowledge and information to make good decisions and then perform a series of actions in a continuous feedback process [33]. In case of an UFDC, however, to perform an action, the drivers need to be aware of the unfamiliar aspect of each new and different driving scenario and quickly process that scenario using the available real-time information. Therefore, it is important to measure the driver's SA when driving under UFDC. Due to already high level of cognitive load in driving under an UFDC, measuring SA should not further increase the driver's cognitive load. Measuring SA by observing the driving performance does not distract the driver and as a result driver's cognitive load will not increase. Individuals with high SA are expected to perform fewer errors in the tasks targeted.

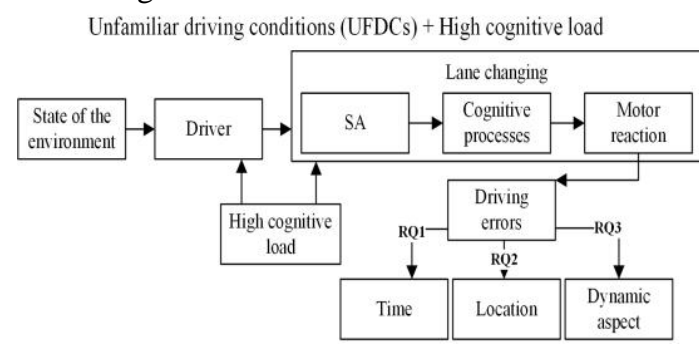

Figure 2. Research model.

Due to the rich dynamics of driving tasks, drivers' SA has to be continuously updated. As the international drivers move through each UFDC scenario, they do not need to know everything. However, they must identify every important piece of information related to the goal of the task. In our study, the target task is to perform a safe lane change. As discussed in the previous sections, existing research has been limited in the assessment of performing the lane changing task when driving in any UFDC associated with high cognitive load. Designing a system based on SA assessment will allow drivers to make correct decisions and thus to take appropriate and safe actions when they perform lane changing tasks in an UFDC. In order to do so, we should adequately address when, where, and why the feedback regarding important information should be presented to the driver by the system for driver-assistance.

Our research model is an adapted version of Endsley [25] (see Figure 2). We will study the driving errors in performing lane changing tasks. Particularly, we will extract the temporal aspects of SA, time, location and dynamic aspects of the errors. These will assist us to draw a list of recommendations to design an efficient SA system (SAS) which considers the drivers' cognitive load.

\section{Research Methodology}

\subsection{Driving Simulator and Driving Scenario}

To answer the research question discussed above, we designed an experiment that could be conducted on a Forum8 UC-win/Road drive simulator [34] at the Simulation Hub, Macquarie University (see Figure 3). The Forum8 UC-win/Road Drive Simulator allows drivers to perform a set of driving tasks and collects the data about a range of driving behaviours. It is an integrated package combining virtual reality software with driving simulator hardware. Software allows the researcher to easily create and edit a range of scenarios including road alignment, visual effects, driving environment, complicated road structures, road crossings and traffic setups. The hardware includes a driving seat with a safety seat belt, steering wheel, accelerator and brake pedal. The front window of the vehicle has been replaced with three monitors displaying the central and peripheral visual fields to the driver as seen in Figure 3.

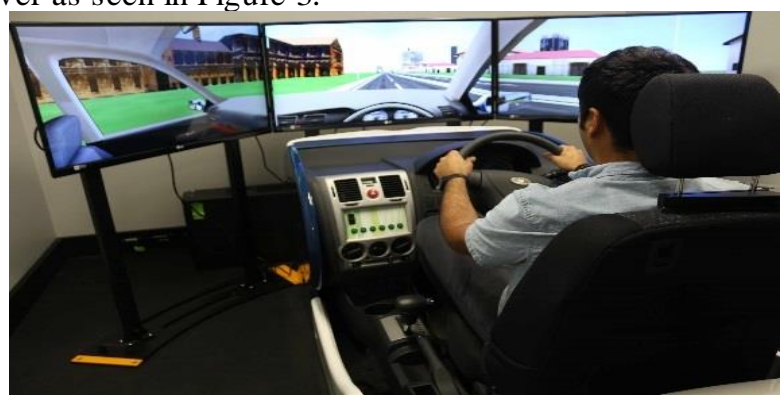

Figure 3. Forum 8 driving simulator.

The driving scenario used in the experiment was designed to test driving skills in a lane changing scenario. All roads were dual-lane with a maximum speed limit, direction, roundabouts and intersection signs. There were no traffic lights, traffic movements or hazards which meant that the participants were able to focus on driving without distractions.

The driving scenario starts with the point $\mathrm{S}_{0}$ which represents the initial start point of the session, whereas 
the point $S_{1}$ represents the start point for recording the data (see Figure 4). The driving session was not recorded from the initial start point to give drivers time to adapt the changing circumstances.

The driving track had three connected road aspects:

1. A straight road section (SR) with a total length $0.716 \mathrm{~km}$ and maximum speed limit of $80 \mathrm{~km} / \mathrm{h}$.

2. A curved section (CR) with $1.313 \mathrm{~km}$ length and maximum speed limit of $40 \mathrm{~km} / \mathrm{h}$.

3. A network of straight roads (NT) has a set of straight roads (NS) that crosses each other in three intersections (Int) and three 4-exit roundabouts (Ra).The roads between the intersections and roundabouts are straight roads. The total length of this network is $1.634 \mathrm{~km}$ with $50 \mathrm{~km} / \mathrm{h}$ maximum speed limit.

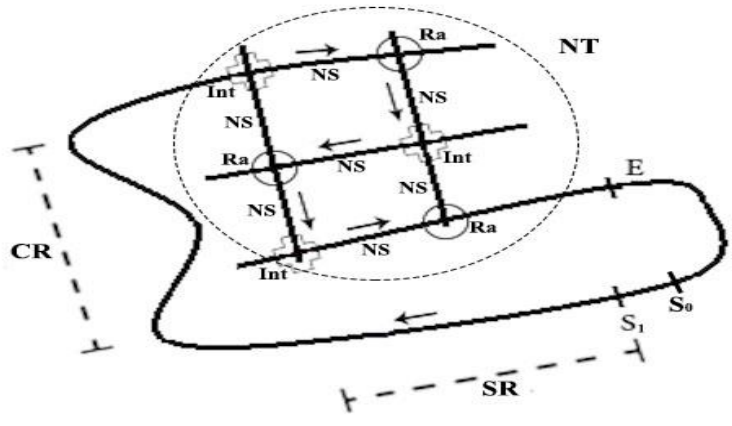

Figure 4. A map of track driving.

\subsection{Sample, Procedure and Tasks}

The study involved 23 participants. The participants ranged in age from 20 to 35 years with a mean age of 25.3 years $(\mathrm{SD}=4.7)$. The participants were selected out of a larger group (i.e. 40 participants) based on the familiarity of traffic system and vehicle configurations. All 23 participants were unfamiliar with the Australian driving conditions (i.e. left-handed traffic and righthand drive vehicle) and they came from different cultural backgrounds. They were familiar with driving in right-handed traffic rules using a left-hand drive vehicle. They had a driving license issued in their home country and a mean driving experience of 6.4 years ( $\mathrm{SD}=4.7)$. Participants drove in familiar conditions for an average 15.7 hours/week (SD = 12).

Participants participated in three sessions: preexperiment, preparation and the driving test. Participants in the pre-experiment session filled out an initial questionnaire regarding their demographic information and driving experience. In the preparation session, participants received verbal instructions about the following session as well as driving rules of lefthanded traffic rules. The next step in the preparation session was getting familiar with the driving test. Participants drove for around 10 minutes in a different scenario to become familiar with the driving simulator. Finally, in the driving test, participants first received a map of the driving scenario supported by the target destination (Figure 4). Participants took around three minutes to study the map. Then, they were asked to perform the main task of the experiment and complete the test with no driving errors. This session ended by asking the participants about the difficulties they faced while driving.

The main task in the driving test was to safely perform necessary lane changing (SNLC), in other words to avoid performing:

1. Unsafe necessary lane changing (UNLC) and

2. Unnecessary lane changing, either safe (SULC) or unsafe (UULC).

In our scenario, the driver was familiar with performing the lane changing task in right-handed traffic using a left-handed driving vehicle. In an UFDC (i.e. left-handed traffic and a right-handed driving vehicle), to make safe necessary lane changing (SNLC), the drivers should follow the following steps:

1. Drivers should keep the vehicle in a slow lane (i.e. the left lane instead of the right lane).

2. Drivers should be aware of cases they need to change the vehicle position at.

3. To make safe lane changing, drivers should be aware of using a proper signal indicator when they depart from their current lane (i.e. the turn indicator stalk of the right-handed vehicle is located on the right of steering wheel instead of the left side of steering wheel).

Lane changing when not needed was classified as "unnecessary lane changing". It might be either safe (SULC) or unsafe (UULC). Considering that there were no traffic movements or other traffic objects on the roads in our experiment, Table 1 illustrates all scenarios of lane changing from left-to-right whereas Table 2 lists all scenarios of lane changing from rightto-left.

Therefore, in our context, drivers were considered to have a high SA if they are able to successfully perform only a safe necessary lane change.

\section{Data Analysis and Results}

The driving simulator of this study, Forum8, generated a $\log$ file of each driver's history. The log file includes a wide range of items that correspond to various driving behaviours. As our study focused on the lane changing task, we selected only data related to that task. More specifically, we looked at:

- Lane number: to indicate the current lane the driver drives in. In our experiment, lane 1 means the left lane and lane 2 means the right lane. 
- Light state: to indicate which turn indicator was used (left or right indicator) or if it was not used (null value).

- Intersection: to recognize the intersections and roundabouts of the road, which represent the need to perform the lane changing task in our experiment.

- Speed in kilometers per hour: to know the dynamic aspect of the situation when the task is performed.

Table 1. Lane changing from left to right.

\begin{tabular}{|c|c|c|}
\hline \multicolumn{2}{|l|}{ Case } & \multirow[t]{2}{*}{ Result } \\
\hline Indicator & Scenario & \\
\hline -Right & $\begin{array}{l}\text {-Upon reaching the second- or third- } \\
\text { exit roundabout. } \\
\text {-Upon reaching the right-turn } \\
\text { intersection. }\end{array}$ & SNLC \\
\hline $\begin{array}{l}\text {-Left } \\
\text {-Not used }\end{array}$ & $\begin{array}{l}\text {-Upon reaching the second- or third- } \\
\text { exit roundabout. } \\
\text {-Upon reaching the right-turn } \\
\text { intersection. }\end{array}$ & UNLC \\
\hline -Right & $\begin{array}{l}\text {-No object at the front. } \\
\text {-Upon reaching the first-exit } \\
\text { roundabout. } \\
\text {-Upon reaching the left-turn } \\
\text { intersection. } \\
\text {-Inside roundabouts or intersections. }\end{array}$ & SULC \\
\hline $\begin{array}{l}\text {-Left } \\
\text {-Not used }\end{array}$ & $\begin{array}{l}\text { No object at the front. } \\
\text {-Upon reaching the first-exit } \\
\text { roundabout. } \\
\text {-Upon reaching the left-turn } \\
\text { intersection. } \\
\text {-Inside roundabouts or intersections. }\end{array}$ & UULC \\
\hline
\end{tabular}

Table 2. Lane changing from right to left.

\begin{tabular}{|c|c|c|}
\hline \multicolumn{2}{|l|}{ Case } & \multirow[t]{2}{*}{ Result } \\
\hline Indicator & Scenario & \\
\hline -Left & $\begin{array}{l}\text {-No object at the front. } \\
\text {-Upon reaching the first- or second- } \\
\text { exit roundabout. } \\
\text {-Upon reaching the left-turn or } \\
\text { straight intersection. }\end{array}$ & SNLC \\
\hline $\begin{array}{l}\text {-Right } \\
\text {-Not used }\end{array}$ & $\begin{array}{l}\text {-No object at the front. } \\
\text {-Upon reaching the first- or second- } \\
\text { exit roundabout. } \\
\text {-Upon reaching the left-turn or } \\
\text { straight intersection. }\end{array}$ & UNLC \\
\hline -Left & $\begin{array}{l}\text {-Upon reaching the third-exit } \\
\text { roundabout. } \\
\text {-Upon reaching the right-turn } \\
\text { intersection. } \\
\text {-Inside roundabouts or intersections. }\end{array}$ & SULC \\
\hline $\begin{array}{l}\text {-Right } \\
\text {-Not used }\end{array}$ & $\begin{array}{l}\text {-Upon reaching the third-exit } \\
\text { roundabout. } \\
\text {-Upon reaching the right-turn } \\
\text { intersection. } \\
\text {-Inside roundabouts or intersections. }\end{array}$ & ULC \\
\hline
\end{tabular}

\subsection{Results}

Overall, 23 participants made 149 lane changes, only 18 lane changes (12\%) were accurate (i.e. safe and necessary), (left-to-right: 11; right-to-left: 7). 131 lane changes $(88 \%)$ were performed with errors, namely UNLC, SULC and UULC (see Figure 5).

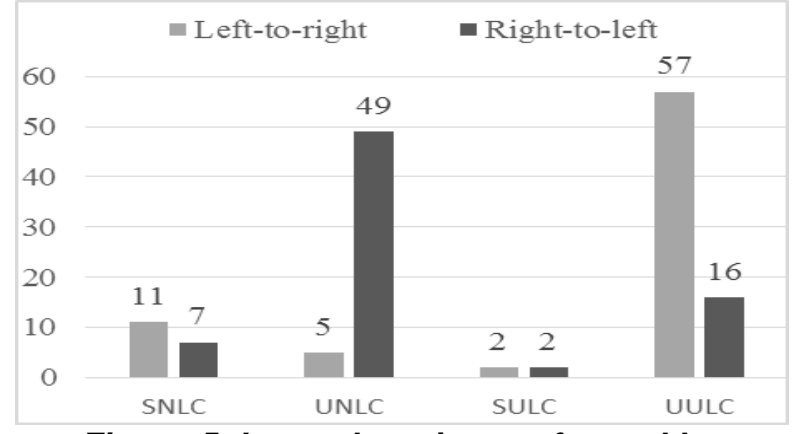

Figure 5. Lane changing performed by participants.

Indicating the lane the driver was planning to depart into was a common error when performing lane changing either from left-to-right or right-to-left. Participants did not manage to use the correct turn indicator when they unnecessarily changed the vehicle's position from left to right lane with 57 UULC out of 64 left-to-right lane changing errors (89\% of left-to-right lane changing errors) (see Table 3). Also participants did not use the turn indicator when they necessarily changed the vehicle's position from right to left lane (49 UNLC out of 67 right-to-left lane changing errors, $73 \%$ ).

Based on the section of the road, errors of performing lane changing occurred at all road sections of the scenario, straight road (SR), curved road (CR) and roads network (NT), see Figure 6. Participants were more likely to make errors in section (NT), 83 errors $(63 \%)$ out of total lane changing errors, from left-to-right (41 errors) and from right-to-left (42 errors). Participants did fewer errors in section (SA) with only 4 errors (3\% of all lane changing errors).

All left-to-right lane changing errors at sections SR and CR were UNLC (see Figure 7). At section NT, UNLC represented the majority of left-to-right lane changing errors by $57 \%$ ( 24 out of 42 left-to-right lane changing errors at section NT). On the other hand, UULC was the only right-to-left lane changing error occurred at sections SR and CR. 59 right-to-left lane changing errors $(88 \%)$ at section NT were UULC.

\subsection{Errors in Roads Network (NT)}

As the section NT had significant number of lane changing errors, we studied the errors of this section in 
more details. The number of lane changing errors at section NT was 83 . We broke down this section into three subsections: intersection (Int), roundabout $(\mathrm{Rb})$ and straight roads in the roads network (SN) subsection. The errors of subsections (Int) and ( $\mathrm{Rb})$ occurred:

- Immediately before reaching intersection/roundabout (BInt/BRb).

- Inside intersection/roundabout (IInt/IRb).

- Immediately after leaving intersection/roundabout (AInt/ARb).

Table 3. The number of lane changing errors.

\begin{tabular}{|l|l|l|l|l|}
\hline $\begin{array}{l}\text { Lane } \\
\text { changing }\end{array}$ & Errors & UNLC & SULC & UULC \\
\hline $\begin{array}{l}\text { Left-to- } \\
\text { right }\end{array}$ & $\begin{array}{l}\text { Out of 64 } \\
(\%)\end{array}$ & $\begin{array}{l}5 \\
(8 \%)\end{array}$ & $\begin{array}{l}2 \\
(3 \%)\end{array}$ & $\begin{array}{l}57 \\
(89 \%)\end{array}$ \\
\hline $\begin{array}{l}\text { Right-to- } \\
\text { left }\end{array}$ & $\begin{array}{l}\text { Out of 67 } \\
\text { (\%) }\end{array}$ & $\begin{array}{l}49 \\
(73 \%)\end{array}$ & $\begin{array}{l}2 \\
(3 \%)\end{array}$ & $\begin{array}{l}16 \\
(24 \%)\end{array}$ \\
\hline
\end{tabular}

After dividing the NT section, we calculated the number of errors of each subsection compared to the total number of NT section. Although the long straight road (SR) had a few number of lane changing errors, the straight roads of the roads network (SN) had the largest number of errors at the roads network (NT) by $35(42 \%)$, see Figure 8. In section (SR), the participants did not need to make any lane changing, whereas the participants in section (SN) needed to perform lane changing due to the intersections and roundabouts on the roads network.

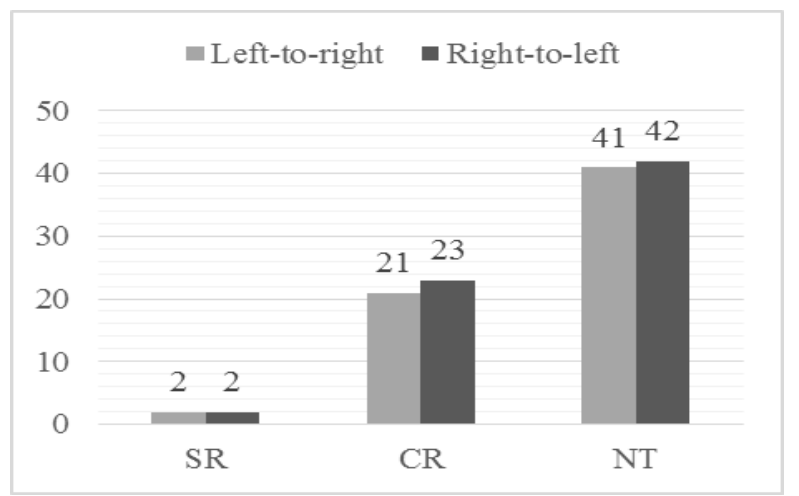

Figure 6. The frequency of lane changing errors on road section: SR, CR and NT.

Also at intersections (Int) and roundabouts (Rb) subsection, participants made a large number of errors, particularly inside intersections (IInt) by $17(21 \%)$, and roundabouts (IRb) by $15(18 \%)$. Also after leaving intersections (AInt) and roundabouts (ARa), participants made some lane changing errors, by 6 (7\%) for each subsection.

\subsection{Speed and Lane Changing}

Driving over the speed limit of SR section was not the reason of the two lane changing errors occurred in that section (see Figure 9). However, over speeding might be a reason of lane changing errors at $\mathrm{CR}$ section, where around $70 \%$ of errors happened by a speed average of $51 \mathrm{~km} / \mathrm{h}$ (the speed limit of CR section is 40). Also around $16 \%$ of lane changing errors associated with over speed $(63 \mathrm{~km} / \mathrm{h})$ in NT section (the speed limit of NT is 50).

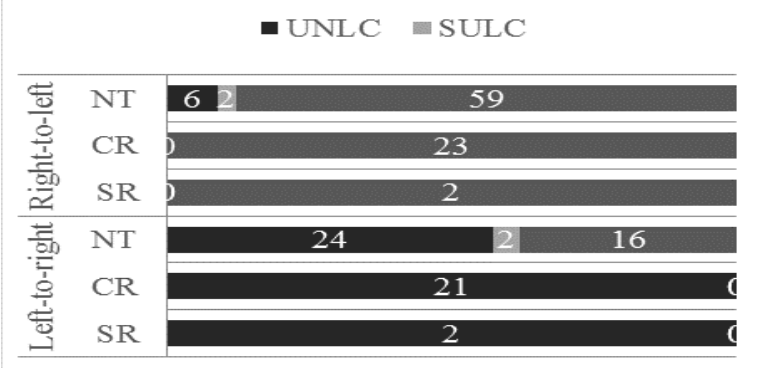

Figure 7. The proportion of making lane changing errors.

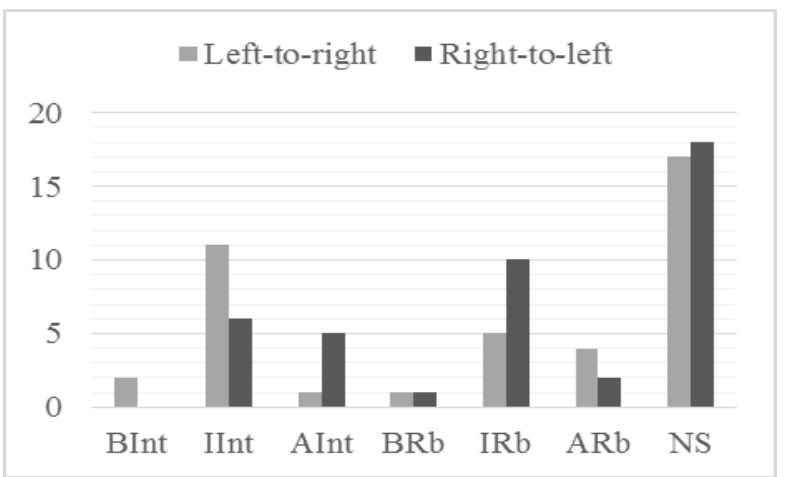

Figure 8. The frequency of lane changing errors in subsections of NS.

\section{Evaluation of Results}

Participants were not able to use the correct turn indicator when they unnecessarily departed from the left to the right lane (UULC) by $89 \%$ of left-to-right lane changing errors. Similar problem appeared with participants when they perform a necessary lane changing (UNLC) from the right to the left lane by $73 \%$ of right-to-left lane changing errors. This might be because of:

- The fact that the driver was unaware of the position of the indicator stalk as the participants were not familiar with the right-handed car configurations,

- Forgetting to use the turn indicator as driving in an UFDC is as sociated with high cognitive load, or 
- Forgetting to use the turn indicator as the participants were not used to use the turn indicator when they drove in their home country.

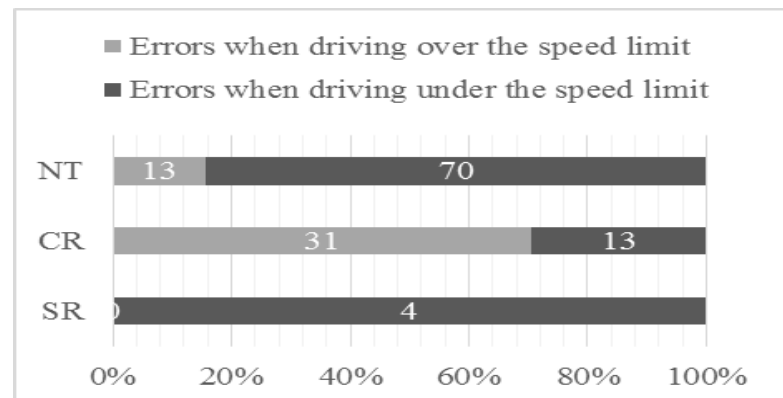

Figure 9. The lane changing errors associated with speeding.

When participants were asked about the difficulty they faced in the driving test, participants P13, P22, and P24 mentioned using the wiper indicator instead of turn indicator as one of difficulties they had. Participant P24 forgot to indicate their lane changing. Participants P4 and P22 stated, "I forgot to use it, I'm not used to it".

Participants experienced more difficulty to perform safe lane changing (SNLC) at NT section. At NT section, the errors were mostly UULC and UNLC when performing left-to-right $(88 \%)$ and right-to-left $(57 \%)$ lane changes respectively.

Overall, participants made more driving errors related to lane changing in NT section (63\%), as this section was more complex than other sections. NT had connections of roads in intersections and roundabouts which might have increased the drivers' cognitive load and as a result increased the error rate at that section.

Also at NT section, participants made lots of errors at SN subsection (42\% of lane changing errors of NT section). This may be because of their cognitive load in complex tasks in addition to unfamiliarity of driving conditions.

Participants were more likely to make lane changing errors at subsections: inside intersections and roundabouts (IInt and IRa respectively) than making lane changing errors at subsections AInt and ARa. Subsections before intersections (BInt) and roundabouts (BRa) had less number of lane changing errors at NT section. These errors might be resulted from the difficulty of the task as $13 \%$ of the participants (P23, P28, P29) mentioned. Moreover, entering intersections and roundabouts requires more information processing as it is different from doing so in familiar driving conditions (i.e. familiar driving conditions for the participants). For instance, in familiar driving conditions, drivers should enter the roundabouts in anticlockwise direction while in an
UFDC (i.e. a right-hand drive vehicle and a left-handed traffic), whilst the drivers should drive through roundabouts in clockwise direction. Participant P29 mentioned that they have never tried that in Australia that is why exiting from first- and third-exit was difficult.

Lane changes were associated with over speeding when making errors at section CR (70\%) but participants were less likely to make lane changing errors in high speed at section NT (16\%). This might be because of the fact that the participants might be unaware of changing the speed limit when they arrived at section CR. The reason for the low level of driver's SA might be the driver's cognitive load being higher at this section, as this section was found very difficult for some participants (22\%). As a result, participants might have made lane changing errors associated with high speed. Participants with code P3, P4, P20, P28 and $\mathrm{P} 40$ mentioned that driving in the curved road was very difficult. Also participant P29 stated, "it was difficult to keep the car at the left side in the curved road". Engstrom et al. [35] stated that cognitive load negatively correlates with the lane keeping variation. Our results conflict with Harms [8] who found that high cognitive load does not affect driving speed. There is need to conduct specific studies to find out the relationship between cognitive load and the rate of lane changing at curved roads.

In NT section, which includes intersections and roundabouts, the participants made less lane changing errors associated with high speed. The complexity of this section might further increase drivers' cognitive load and thus led the participants reduce their speed, particularly when reaching the intersections and roundabouts. These results are aligned with the results of [8] who found that cognitive load inversely correlates with driving speed. In [8], the driver's cognitive load was the highest while approaching and driving through the junctions at the same time of decreasing the driving speed.

\section{Conclusion}

In this paper, Forum8 driving simulator was used to capture the data related to lane changing tasks. 23 participants performed the driving tasks in a simulated scenario. The driving scenario included three main road sections: straight road, curved road and road network. The road network had three subsections: intersections, roundabouts and straight road.

Our results answer the research questions: when, where and why does the driver make errors of the lane changing tasks under an UFDC? 
Based on the components of the situation awareness, the answer to the research question is structured in the following statement and matrix representation in Table 4:

"If driving [time (T): on/before/inside/after] [location (L): section/subsection of the road] with [speed (S): over/under] the speed limit, then the participant has [situation awareness level (SA): low/high] situation awareness".

Table 4. Lane changing error matrix representation

\begin{tabular}{|l|l|l|l|}
\hline T & L & S & SA \\
\hline On & Curved road & Over & Low \\
\hline Inside & Intersections & Under & Low \\
\hline Inside & Roundabouts & Under & Low \\
\hline After & Intersections & Under & Low \\
\hline After & Roundabouts & Under & Low \\
\hline On & Straight road & Under & High \\
\hline Before & Intersections & Under & High \\
\hline Before & Roundabouts & Under & High \\
\hline
\end{tabular}

Also the results showed that participants made a significant number of lane changing errors (88\%) compared to the accurate lane changes (12\%). This may necessitate the development of an augmented feedback system to increase situation awareness, particularly in the cases of low level of situation awareness in Table 4.

As most of lane changing errors in our experiments were associated with not using the turn indicator, recording such reactions would provide an extra objective assessment and help us to get better understanding of driving performance.

We plan to extend our work in two stages. First stage aims to get a better understanding of international drivers' behaviour and it includes the following points:

- Recording the experiments using cameras to get richer information of driving performance.

- Comparing the driving performance between familiar and unfamiliar drivers with the same driving conditions (i.e. left-handed traffic rules and a righthanded vehicle).

- Analyzing the collected data regarding to other driving tasks, such as driving at roundabouts and intersections.

Using the results of this study and the first stage of our future work, the second stage includes the following:

- Using the results of our study, designing a driver assistant system. Using augmented feedback will aim to increase the drivers' situation awareness in high cognitive load driving conditions.

- Drawing comparisons between the driving performance with and without the proposed system as well as comparisons between using the system in a simulator and a real environment.

\section{Acknowledgment}

This study has been sponsored by King Abdulaziz University through a $\mathrm{PhD}$ scholarship to the first author. Authors are thankful to Dr. Stephen Smith for his contribution in Situation Awareness section.

\section{References}

[1] Sweller, J., J.J. Van Merrienboer, and F.G. Paas, Cognitive architecture and instructional design. Educational psychology review, 1998. 10(3): p. 251-296.

[2] Page, S.J. and D. Meyer, Tourist accidents: an exploratory analysis. Annals of Tourism Research, 1996. 23(3): p. 666-690.

[3] Wilks, J., B. Watson, and J. Hansen, International drivers and road safety in Queensland, Australia. Journal of Tourism Studies, 2000. 11(2): p. 36-43.

[4] Tourism Forecasting Committee, Forecast. 2013, Tourism Research Australia: Canberra.

[5] Piao, J. and M. McDonald, Advanced driver assistance systems from autonomous to cooperative approach. Transport Reviews, 2008. 28(5): p. 659-684.

[6] Endsley, M.R. Situation awareness global assessment technique (SAGAT). in Aerospace and Electronics Conference, 1988. NAECON 1988., Proceedings of the IEEE 1988 National. 1988. IEEE.

[7] Endsley, M.R., A survey of situation awareness requirements in air-to-air combat fighters. The International Journal of Aviation Psychology, 1993. 3(2): p. 157-168.

[8] Jin, S. and D. Kaber. The role of driver cognitive abilities and distractions in situation awareness and performance under hazard conditions. in proceedings of the IEA 2009 17th World Congress on Ergonomics, Beijing, China. 2009.

[9] Roscoe, S. and R. North, Prediction of pilot performance. Aviation psychology.(A 81-15676 04-53) Ames, Iowa State University Press, 1980, 1980: p. 127-133.

[10] Strater, L.D., et al., Measures of platoon leader situation awareness in virtual decision-making exercises. 2001, DTIC Document.

[11] Taylor, R., Situational Awareness Rating Technique(SART): The development of a tool for aircrew systems design. AGARD, Situational Awareness in Aerospace Operations 17 p(SEE N 90-28972 23-53), 1990. 
[12] Yale, S.H., et al., Neurologic conditions: assessing medical fitness to drive. Clinical medicine \& research, 2003. 1(3): p. 177-188.

[13] Holahan, C.J., R.E. Culler, and B.L. Wilcox, Effects of visual distraction on reaction time in a simulated traffic environment. Human Factors: The Journal of the Human Factors and Ergonomics Society, 1978. 20(4): p. 409-413.

[14] Gregoriades, A., et al., Human-centered safety analysis of prospective road designs. Systems, Man and Cybernetics, Part A: Systems and Humans, IEEE Transactions on, 2010. 40(2): p. 236-250.

[15] Pradhan, A.K., et al., Using eye movements to evaluate effects of driver age on risk perception in a driving simulator. Human Factors: The Journal of the Human Factors and Ergonomics Society, 2005. 47(4): p. 840-852.

[16] Palinko, O., et al. Estimating cognitive load using remote eye tracking in a driving simulator. in Proceedings of the 2010 symposium on eye-tracking research \& applications. 2010. ACM.

[17] Mohri, Y., et al. Physiological magnetic stimulation on car driver's spine for arousal preventing drowsy driving without rebound sleep and back magnetocardiogram. in Proceedings of the IEEE International Magnetics Conference (INTERMAG'15). 2015.

[18] Medenica, Z., et al. Augmented reality vs. street views: a driving simulator study comparing two emerging navigation aids. in Proceedings of the 13th International Conference on Human Computer Interaction with Mobile Devices and Services. 2011. ACM.

[19] Denoual, T., et al., Drivers' perception of loss of adherence in bends: influence of motion rendering. Journal of Computing and Information Science in Engineering, 2011. 11(4): p. 041004.

[20] Uchiyama, Y., et al., Suppression of brain activity related to a car-following task with an auditory task: An fMRI study. Transportation research part F: traffic psychology and behaviour, 2012. 15(1): p. 25-37.

[21] Mahr, A., et al., The contre (continuous tracking and reaction) task: A flexible approach for assessing driver cognitive workload with high sensitivity. Automotive user interfaces and interactive vehicular applications, 2012: p. 8891.

[22] Saito, S., et al. An International Driving Simulator: Recognizing the Sense of a Car Body by the Simulator. in Advanced Information Networking and Applications Workshops (WAINA), 2012 26th International Conference on. 2012. IEEE.
[23] Drews, F.A., et al., Text messaging during simulated driving. Human Factors: The Journal of the Human Factors and Ergonomics Society, 2009.

[24] Endsley, M.R., A taxonomy of situation awareness errors. Human factors in aviation operations, 1995. 3(2): p. 287-292.

[25] Stanton, N.A., A. Dunoyer, and A. Leatherland, Detection of new in-path targets by drivers using Stop \& Go Adaptive Cruise Control. Applied ergonomics, 2011. 42(4): p. 592-601.

[26] Salmon, P.M., et al., Measuring Situation Awareness in complex systems: Comparison of measures study. International Journal of Industrial Ergonomics, 2009. 39(3): p. $490-500$.

[27] Lee, J.D., et al., Speech-based interaction with invehicle computers: The effect of speech-based e-mail on drivers' attention to the roadway. Human Factors: The Journal of the Human Factors and Ergonomics Society, 2001. 43(4): p. 631-640.

[28] Rakauskas, M.E., L.J. Gugerty, and N.J. Ward, Effects of naturalistic cell phone conversations on driving performance. Journal of safety research, 2004. 35(4): p. 453464.

[29] Jamson, A.H., et al., Speech-based e-mail and driver behavior: Effects of an in-vehicle message system interface. Human Factors: The Journal of the Human Factors and Ergonomics Society, 2004. 46(4): p. 625-639.

[30] Panou, M., E. Bekiaris, and V. Papakostopoulos, Modelling Driver Behaviour in European Union and International Projects, in Modelling driver behaviour in automotive environments. 2007, Springer. p. 3-25.

[31] Wang, J., K. Li, and X.-Y. Lu, Effect of Human Factors on Driver Behavior. 2014.

[32] Lunenfeld, H. Human factor considerations of motorist navigation and information systems. in Vehicle Navigation and Information Systems Conference, 1989. Conference Record. 1989. IEEE.

[33] FORUM8. UC-win/Road [Online]. FORUM8. Available: http://www.forum8.co.jp/english/uc-win/ucwinroad-e1.htm [Accessed May 6, 2016].

[34] Engström, J., E. Johansson, and J. Östlund, Effects of visual and cognitive load in real and simulated motorway driving. Transportation Research Part F: Traffic Psychology and Behaviour, 2005. 8(2): p. 97-120.

[35] Harms, L., Variation in drivers' cognitive load. Effects of driving through village areas and rural junctions. Ergonomics, 1991. 34(2): p. 151-160. 